\title{
PROPOSALS FOR AN IMPROVED NUTATION FORMULA
}

\author{
E. GROTEN, S.Y. ZhU * \\ Institute Physical Geodesy \\ Petersenstrasse 13 \\ D-6100 Darmstadt \\ Germany, Federal Republic \\ * on leave from Shanghai Observatory, Shanghai, China
}

There are a variety of reasons why in geodesy an improved formula for nutation is needed; related topics of interest are the determination of time-dependences in low degree zonals of the earth gravity field, ocean tide modeling, determination of odd harmonics of gravity field etc. in satellite geodesy. A combined model of deterministic and stochastic components is used in order to evaluate two new nutation series where, in an adjustment, mainly VLBI data (IRIS, GSFC, IERS) have been applied. Contrary to earlier revisions of the present nutation formula, not only the five significantly affected waves (annual, semi-annual, FCN etc.) are corrected but rather all constituents are revised in such a way that white noise residuals result from the adjusted observations, based on the new formula. Still remaining problems (such as the separation of long-period terms from precession etc.) are outlined.

Alternatives to the approach applied in (Zhu et al., 1989) have been considered; one possibility would be to dispose otherwise of the FCN-period, differently from the aforementioned paper. However, due to the still inherent uncertainties in polar motion etc. at this time, a substantial change in comparison to the aforementioned paper does not seem to be available now. Consequently, also a solution for the free core nutation period $(\mathrm{FCN})$ does not yet appear appropriate with the existing VLBI-data at hand.

\section{References}

Zhu, S.Y., E. Groten and Ch. Reigber, "Various aspects of numerical determination of nutation constants part II", to appear in Astron. J., 1989. 